\title{
SURVEY OF SOFT BIOMETRIC TECHNIQUES FOR GENDER IDENTIFICATION
}

\author{
ANKUSH RAI*, JAGADEESH KANNAN R
}

School of Computer Science \& Engineering, VIT University, Chennai, Tamil Nadu, India. Email: ankushressci@gmail.com

Received: 13 December 2016, Revised and Accepted: 07 April 2017

\begin{abstract}
Biometrics checks can be productively utilized for localization of intrusion in access control systems by utilizing soft computing frameworks. Biometrics procedures can be to a great extent separated into conventional and soft biometrics. The study presents a survey of the available soft techniques and comparison for gender identification from biometric techniques.
\end{abstract}

Keywords: Soft computing, Biometric images, Gender identification.

(C) 2017 The Authors. Published by Innovare Academic Sciences Pvt Ltd. This is an open access article under the CC BY license (http://creativecommons. org/licenses/by/4. 0/) DOI: http://dx.doi.org/10.22159/ajpcr.2017.v10s1.19741

\section{INTRODUCTION}

Introduction to biometric gender identification

The concept of fingerprint pattern being studied has been of significant use over time, once scanning it involves the conversion of fingerprint by tiny portion of light solid-state devices into alphanumeric formula.

A fingerprint is that the representation of the epidermis of a finger; it consists of a pattern of interleaved ridges and valleys. Fingertip ridges evolved over the years to permit humans to understand and grip objects. Like everything within the human body, fingerprint ridges form through a mix of genetic and environmental factors.

The technology of using human body for identification purpose is understood as "biometrics," the word was derived from Greek word sense bio for "life" and metric for "measurement," the authentication of biometrics for private identification is extremely and a lot of reliable compared with one thing you recognize which might be forgotten (such as password and registration numbers) or what you have got (such as identity card, physical lock, and smart card) which might be misplaced compared with one thing you're or element of your body [1].

Biometrics measures the distinctive physical or behavioral characteristics of individual as a method to recognize or authenticate their identity. Common physical biometrics includes fingerprints, hand or palm geometry, and retina, iris or facial characteristics. Behavioral characteristics such as signature, voice (which additionally features a physical component), keystroke pattern, and gait. Although some technologies have gained access control and biometrics as full shows great potential to be used in end-user segments, such as airports, stadiums, defense installations and therefore the industry and corporate workplaces wherever security and privacy are needed $[2,3]$.

Recently, there has been associate degree accumulated interest in biometric technologies that's human identification supported one's individual features the varied identification data used are fingerprints, handwriting, bite marks, DNA fingerprinting, etc. Fingerprints are constant and individualistic and kind the foremost reliable criteria for identification. Fingerprints are one in every of the foremost mature biometric technologies and are thought of legitimate proofs of evidence in courts of law everywhere the world. Supported the styles of information available from the fingerprint, we have a tendency to are ready to method its identity alongside gender, age and quality at interval today atmosphere of the accumulate importance of security and organization, identification, and authentication methods have developed into a key technology. Such demand for reliable personal identification in processed access management has resulted within the accumulated interest in biometrics. Fingerprint identification algorithms are well established and are being implemented everywhere the world for security and person identity. Only a few attempts associate degree attempt are created to classify the gender from an obtained fingerprint this can be useful for anthropologists for classifying gender from the fingerprints they get from excavated articles and for crime investigators for minimizing the trend of the suspects. The gender of the person is judged using the fingerprint of that concern person based mostly on the count of the ridges of the fingerprint. The average ridge count is slightly higher in males than in females, with high variance among subjects of each gender. Gender classification is a crucial downside with a spread of practical applications [4]. For instance, a strong gender classification might offer a basis for acting passive police investigation using demographic information or grouping valuable consumer statistics in a very shopping center. It might even be wont to improve the performance of biometric systems such as face authentication and recognition. In computer vision, the bulk of studies on gender classification is supported face since visual information from human faces provides necessary cues for gender classification. Supported the styles of the information available from the fingerprint, we have a tendency to are ready to method its identity along with gender, age and quality.

\section{Motivation}

Gender classification from fingerprints is a crucial step in forensic science so as to produce investigatory leads for locating unknown persons. Fingerprint identification algorithms are well established and are being enforced everywhere the world for security and person identity. Only a few attempts associate degree attempt are created to classify the gender from an obtained fingerprint this can be useful for anthropologists for classifying gender from the fingerprints they get from excavated articles and for crime investigators for minimizing the trend of the suspects. The gender of the person is judged using the fingerprint of that concern person based mostly on the count of the ridges of the fingerprint. The average ridge count is slightly higher in males than in females, with high standard deviation among subjects of each gender. Gender classification is a crucial downside with a spread of practical applications. For example, a strong gender organization might offer a basis for performing passive surveillance using demographic information or grouping valuable consumer statistics in a very shopping center. It might even be used to improve the performance of biometric systems such as face authentication and recognition. In computer vision, the bulk of studies on gender classification is supported on the 
face since visual information from human faces provides necessary cues for gender classification. Supported the styles of the information available from the fingerprint, we have a tendency to are ready to method its identity alongside gender, age, and quality.

We have planned a way for gender classification which can use some features of finger-like ridge thickness, ridge thickness to valley thickness ratio (RTVTR) and ridge mensuration for gender detection.

\section{Multi-class support vector machines (SVMs)}

Classifying data could be a common task in machine learning. Suppose some given data points every belong to one of two categories, and therefore the goal is to choose that category a new data point will be in. In the case, SVMs are used. In machine learning, SVMs (additionally support vector networks) are supervised learning models with associated learning algorithms that analyze data and acknowledge patterns, used for classification and multivariate analysis. Given a group of training examples, every marked as happening to one of the two categories, an SVM training algorithm rules builds a model that assigns new examples into one class or the opposite, creating it a non-probabilistic binary linear classifier [5-7]. An SVM model could be an illustration of the examples as points in space, mapped so the sample of the separate classes is divided by a transparent gap that's as wide as possible. New examples are then mapped into that very same space and expected to belong to a class supported that aspect of the gap they fall on.

More formally, a SVM constructs a hyperplane or set of hyperplanes in a very high- or infinite dimensional space, which might be used for classification, regression, or different tasks. Intuitively, a decent separation is achieved by the hyperplane that has the biggest distance to the closest training data point of any category (so-called functional margin) since normally the larger the margin the lower the generalization error of the classifier.

Whereas the first downside is also expressed in a very finite dimensional space, it usually happens that the sets to discriminate are not linearly severable therein that space. For this reason, it absolutely was planned that the first finite dimensional space is mapped into a far higherdimensional space, presumptively creating the separation easier in that space. To stay the process load cheap, the mappings utilized by SVM schemes are designed to make sure that dot products are also computed simply in terms of the variables within the original space, by process them in terms of a kernel function $\mathrm{K}(\mathrm{x}, \mathrm{y})$ selected to suit the matter [8].

Unfortunately, unlike KDA, SVMs do not generalize naturally to the multi-class classification case. SVMs are binary classifiers, and in and of its, in their original kind, they'll solely decide between two categories directly. However, several approaches are steered to perform multi-class classification using SVMs, one in every which can be exemplified below. Suppose we have to choose between three categories, A, B, and C. Now, suppose all we've are binary classifiers, i.e., ways which might mechanically decide only solely between two categories. A potential approach so as to use our binary classifiers in a very multi-class classification downside would do to divide our multiclass downside into a group of binary problems. The left matrix below shows all potential combinations of binary decision problems which might be formed by taking our three categories [9]

\section{LITERATURE SURVEY}

\section{Survey}

Fingerprint identification and classification have been extensively researched in times past; however, very few researchers have studied the fingerprint gender classification problem.

In [10] authors have proposed a method for fingerprint-based gender classification through frequency domain analysis to estimate gender by analyzing fingerprints using two-dimensional discrete wavelet transforms and principal component analysis. A dataset of 400 persons of different age and gender is collected as internal database. They have used minimum distance method for classification and achieve overall success rate in gender classification of around $70 \%$.

In [11] researchers have worked on fingerprint-based gender identification using frequency domain analysis. The classification is achieved by analyzing fingerprints using fast Fourier transform, discrete cosine transform, and power spectral density. A dataset of 220 persons of different age and gender is collected as internal database. Frequency domain calculations are compared with predetermined threshold and gender is determined. They obtained results of $90 \%$ and $79.07 \%$ for female and male samples, respectively.

Authors of Valarmathy and Kumar [12] in their study provided an aid for the fingerprint examiner in analyzing fingerprint samples as it shows that there is a significant difference in epidermal ridge density between males and females of the two populations. Their study has been carried out to examine ridge density differences in two Northern Indian populations (Sikh Jat and Bania). In their study, it has been found that $92 \%$ of Sikh Jat females have a mean ridge density above 13 , whereas $76 \%$ of Sikh Jat males have (a mean ridge density) below 13 , while in Bania, $100 \%$ of females have mean ridge density above 14 and $80 \%$ of males - below 14 . The study suggested that there are significant differences in epidermal ridge density between males and females within each of the two populations and also significant differences between the two populations.

In [13] the authors worked on gender determination using fingertip features. He obtained fingerprints from 115 normal healthy adults in which 57 were male fingerprints and 58 were female fingerprints. They have used ridge count, ridge density, and finger size features for classification. However, the ridge count and finger size features of left little fingers are used to achieve a classification. The best classification result of $86 \%$ accuracy is obtained using ridge count and finger size feature together.

Researchers of Singh et al. [14] proposed a method for gender classification from fingerprints. Features extracted were ridge width, RTVTR, and ridge density. SVM is used for the classification. This method is experimented with the internal database of 400 fingerprints in which 200 were male fingerprints and 200 were female fingerprints. They found male - female can be correctly classified up to $91 \%$.

In [15] they had proposed a gender classification from fingerprints, which is an important step in forensic anthropology to identify the gender of a criminal and minimize the list of suspects search. A dataset of 10 - fingerprint images for 2200 persons of different ages and gender (1100 males and 1100 females) was analyzed. Features extracted were ridge count, RTVTR, white lines count, and ridge count asymmetry, and pattern type concordance. Fuzzy C-means (FCM), linear discriminant analysis (LDA), and neural network (NN) were used for the classification using the most dominant features. They obtained results of $80.39 \%$, $86.5 \%$, and $88.5 \%$ using FCM, LDA, and NN, respectively.

In this [16] study, it has been presented a study whose aim is to determine if women have significantly higher ridge density, hence finer epidermal ridge detail, than men by counting ridges that occur within a well-defined space. If significant gender differences do exist then the likelihood of inferring gender from given ridge densities will be explored. Their study focused on 400 randomly picked 10-print cards representing 400 subjects. The demographic composition of this sample population represents 100 Caucasian males, 100 African American males, 100 Caucasian females, and 100 African American females all within the age range of 18-67. Results show that women tend to have a significantly higher ridge density than men and that this trend is upheld in subjects of both Caucasian and African American descent $(\mathrm{F}=81.96, \mathrm{p}<0.001)$. Application of Bayes' theorem suggests that a given fingerprint possessing a ridge density of 11 ridges $/ 25 \mathrm{~mm}^{2}$ or less is most likely to be of male origin. Likewise, a fingerprint having a ridge density of 12 ridges $/ 25 \mathrm{~mm}^{2}$ or greater is most likely to be of female origin, regardless of race. 
In the study [17] it was presented that there is an association between distribution of fingerprint patterns, blood group, and gender. This prospective study was carried out over a period of 2-month among 200 medical students (100 male and 100 female) belonging to the age group 18-25 of Kasturba Medical College, Mangalore, India. Results show that each fingerprint is unique; loops are the most commonly occurring fingerprint pattern while arches are the least common. Males have a higher incidence of whorls and females have a higher incidence of loops. Loops are predominant in blood group $\mathrm{A}, \mathrm{B}, \mathrm{AB}$ and $\mathrm{O}$ in both $\mathrm{Rh}$ positive and $\mathrm{Rh}$ negative individuals except in $\mathrm{O}$ negative where whorls are more common. Thus, they concluded that there is an association between distribution of fingerprint patterns, blood group, and gender and thus the prediction of gender and blood group of a person is possible based on his fingerprint pattern.

\section{Overview of fingerprinting}

Human fingerprints have been discovered on a large number of archeological artifacts and histological items. Although these findings provide evidence to show that ancient people were aware of the individuality of fingerprints, it was not until the late $16^{\text {th }}$ century that the modern scientific fingerprint technique was first initiated in Ramotowski et al. [18]. In 1686, Marcello Malpighi, a professor of anatomy at the University of Bologna noted in his writings the presence of ridges, spirals and loops in fingerprints. Since then, a large number of researchers have invested huge amounts of effort on fingerprint studies. Henry Fauld, in 1880, was the first to scientifically suggest the individuality of fingerprints based on an empirical observation. At the same time, Herschel asserted that he had practiced fingerprint recognition for about 20 years [19]. These findings established the foundation of modem fingerprint recognition. In the late nineteenth century, Sir Francis Galton conducted an extensive study on fingerprints; he introduced the minutiae features for fingerprint matching in 1888. An important advance in fingerprint recognition was made in 1899 by Edward Henry, who established the well-known "Henry system" of fingerprint classification. In the early 20th century, fingerprint recognition was formally accepted as a "valid personal identification method and became a standard routine in forensics (Lee et al. 2001)." Fingerprint identification agencies were set up worldwide and criminal fingerprint databases were established [20-24]. Various fingerprint recognition techniques - including latent fingerprint acquisition, fingerprint classification, and fingerprint matching - were developed. For example, the FBI fingerprint identification division was set up, in 1924, with a database of 810,000 fingerprint cards (Federal et al. 1984). European explorer Joao de Barros recorded the first known example of fingerprinting, which is a form of biometrics, in China during the $14^{\text {th }}$ century. Chinese merchants used ink to take children's fingerprints for identification purposes. In 1890, Alphonse Bertillon studied body mechanics and measurements to help in identifying criminals. The police used his method, the Bertillon age method, until it falsely identified some subjects. The Bertillon age method was quickly abandoned in favor of fingerprinting, brought back into use by Richard Edward Henry of Scotland Yard. Karl Pearson, an applied mathematician studied biometric research early in the 20th century at University College of London. He made important discoveries in the field of biometrics through studying statistical history and correlation, which he applied to animal evolution. His historical work included the method of moments, the Pearson system of curves, correlation and the Chisquared test. In the 1960 s and 70 s, signature biometric authentication procedures were developed, but the biometric field remained fixed until the military and security agencies researched and developed biometric technology beyond fingerprinting [24]. Since 700 AD, this science of fingerprint has been used for the purpose of identification. Chinese used fingerprints as official documents in 3000BC. The system was first used in India in 1858 by Sir William Herschel to prevent impersonation, but the credit is given to Sir Francis Galton for having it systematized for the identification of criminals. His system was officially adopted in England in 1894, and was further modified by Sir Edward Henry. Afterward the studies have been conducted on fingerprint ridges mainly its types, classification, methods of lifting fingerprints, recording of fingerprints, and materials used to develop fingerprint (Gungadin et al. 2007). Fingerprint identification and classification has been extensively researched in times past; however, very few researchers have studied the fingerprint gender classification problem (Acree et al. 1999), used the ridge density, which he defined as the number of ridges in a certain space; it was shown that the females have higher ridge density (Acree et al. 1999). Kralik et al. 2003 also showed that the males have higher ridge breadth, which was defined as the distance between the centers of two adjacent valleys, than females. Two studies showed that the males have higher ridge count than the females (Hall et al. 2003, and Cote et al. 2002. It was shown that both males and females have higher rightward directional asymmetry in the ridge count, with the asymmetry being higher in males than females (Hall et al. 2003), and higher incidence of leftward asymmetry in females (Cote et al. 2002). Female's fingerprints are significantly of lower quality than male fingerprints (Hicklin et al. 2002). While the appearance of white lines and scars in fingerprint images are very common in housewives [25]. By the $7^{\text {th }}$ month of natal development, the dermatoglyphic patterns of the fingers are complete and no further modifications can occur during maturation except in the case of accident. Since, during growth, the overall size of the palm increases, the fingerprint increase in size without adding new ridges and ridge breadth this is defined as the measurement from the center of one furrow across the ridge to the center of the next furrow (Penrose et al. 2001). Within individual, the breadth of fingerprint ridge varies within the hand and between hands, but the difference is quite small, on the order of $0.05 \mathrm{~mm}$ and less (Cummings et al. 1994). Although it has been found that males tend to have more ridge counts than females, inconsistent results have been obtained with regard to the statistical significance of this sex difference. It has also been shown that women tend to have a higher ridge density (ridge counts divided by the size of the corresponding fingertip area) than men but the sex determination accuracy of this feature does not seem to be very satisfactory (Acree et al. 1999). The female's fingerprint is characterized by a high RTVTR, while the male's fingerprint is characterized by low RTVTR, with the exception of small percentage of male's fingerprints having high RTVTR, and female's fingerprints having low RTVTR [26,27]. The female's fingerprint is characterized by high count of white lines, with the exception of small percentage having few or no white lines. The male's fingerprint is characterized by having no or few number of white lines, with the exception of small percentage having high count of white lines also that the male's ridge count is slightly higher than the female's, with high standard deviation in its distribution among both genders, ridge count for the females, with $\mu=13.6671, s=4.9845$, and the males, with $\mu=14.6914, \mathrm{~s}=4.9336$, with $\mathrm{t}$-value $=4.802$, and $\gamma$-value $=1.685 \mathrm{E}-06$ (Badawi et al. 2006). The size of the fingertip has a strong relationship to the values of ridge counts and ridge density. If males have more ridge counts and smaller ridge densities than females, then the finger size difference between males and females should be more significant than the features of ridge count and ridge density (Wang et al. 2008). Gungadin et al. (2007) opined that a ridge count of $=13$ ridges $/ 25 \mathrm{~mm}^{2}$ is more likely to be of male origin and that of $=14$ ridges $/ 25 \mathrm{~mm}^{2}$ is likely to be of female origin. The outcome of his study is that women tend to have more ridge density than men [28].

\section{Fingerprint classification}

Fingerprint identification and verification are one of the most significant and reliable identification methods. It is virtually impossible that two people have the same fingerprint, having a probability $1 / 1.9 \times 1015$ (Hong et al. 2000). In fingerprint identification and verification applications worldwide, a large volume of fingerprints are collected and stored for a wide range of applications, including forensics, civilian, commercial and law-enforcement applications. Automatic identification of humans based on fingerprints requires the input fingerprint to be matched with a large number of fingerprints in a database (for example, the FBI database contains approximately 70 million fingerprints). To reduce the search time and computational complexity, it is desirable to classify the database into accurate and consistent classes so that input fingerprint is matched only with a subset of the fingerprints in the database. The nature of each application will 
determine the degree of accuracy required. For example, a criminal investigation case may require higher degree match than access control case systems. Automatic fingerprint classification methods, such as methods introduced in Drets et al. (1999) rely on point patterns in fingerprints, which form ridge endings and bifurcation unique to each person. Traditionally, activities to solve a pattern recognition task are twofold. First, a set of features has to be found describing the object(s) being classified. Second, after a set of features has been found, a classification mechanism is chosen and optimized. These two steps are highly interdependent, since the choice of features influences the conditions under which a classifier operates, and vice versa [29]. With the advent of NNs however, more and more problems are solved by simply feeding large amounts of "raw data" (e.g., images, sound signals, stock market index ranges) to a NN. This approach, however, is not feasible in fingerprint classification, which are highly susceptible to noise and elastic distortions. Therefore, it is desirable to extract features from the images that are invariant to such distortions. During training the classification network learns the association and significance of features. An attempt has been made previously to study fuzzy logic and artificial NN techniques in fingerprint identification [30].

\section{Fingerprint feature extraction}

The central problem in designing a fingerprint classification system is to determine what features, should be used and how categories are defined based on these features. There are, mainly two types of features that are useful for fingerprint recognition system: (i) Local ridge and valley details (minutiae) which have different characteristics for different fingerprints, and (ii) global pattern configurations, which form special patterns of ridges and valleys in the central region of the fingerprint. The first type of features carries for the information about the individuality of fingerprints and the second type of features carry information about the fingerprint class. Therefore, for fingerprint classification, the features derived from the global pattern configurations should be used. These features should be invariant to the translation and rotation of the input fingerprint images. Generally, global fingerprint features can be derived from the orientation field and the global ridge shape. The orientation field of a fingerprint consists of the ridge orientation tendency in local neighborhoods and forms an abstraction of the local ridge structures. It has been shown that the orientation field is highly structured and can be roughly approximated by the core and delta models which are known as singular points details [31-33].

Fingerprints may be deposited in natural secretions from the eccrine glands present in friction ridge skin or they may be made by ink or other contaminants transferred from the peaks of friction skin ridges to a relatively smooth surface [34]. Fingerprint patterns are genotypically determined and remain unchanged from birth till death. Fingerprints collected at a crime scene can be used to identify suspects, victims and other persons who touched the surface, fingerprint scans can be used to validate electronic registration, cashless catering and library access especially in schools and colleges. The most common ridge characteristics are shown in the Fig. 1.

\section{SVM}

In machine learning, SVMs (also support vector networks) are supervised learning models with associated learning algorithms that analyze data and recognize patterns, used for classification and regression analysis. Given a set of training examples, each marked as belonging to one of the two categories, an SVM training algorithm builds a model that assigns new examples into one category or the other, making it a no probabilistic binary linear classifier. An SVM model is a representation of the examples as points in space, mapped so that the examples of the separate categories are divided by a clear gap that is as wide as possible. New examples are then mapped into that same space and predicted to belong to a category based on which side of the gap they fall on [35].

Classifying data is a common task in machine learning. Suppose some given data points each belong to one of two classes, and the goal is to decide which class a new data point will be in. In the case of SVMs, a

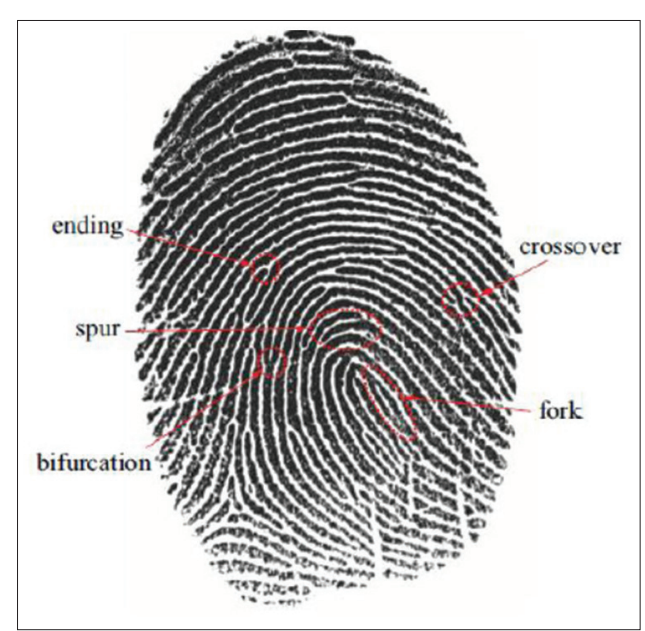

Fig. 1: The most common ridge characteristics

data point is viewed as a p-dimensional vector (a list of p numbers), and we want to know whether we can separate such points with a (p-1)-dimensional hyperplane. This is called a linear classifier. There are many hyperplanes that might classify the data. One reasonable choice as the best hyperplane is the one that represents the largest separation, or margin, between the two classes. So we choose the hyperplane so that the distance from it to the nearest data point on each side is maximized. If such a hyperplane exists, it is known as the maximum-margin hyperplane and the linear classifier it defines is known as a maximum margin classifier; or equivalently, the perceptron of optimal stability.

Potential drawbacks of the SVM are the following three aspects:

- Uncalibrated class membership probabilities

- The SVM is only directly applicable for two-class tasks. Therefore, algorithms that reduce the multi-class task to several binary problems have to be applied

- Parameters of a solved model are difficult to interpret.

\section{Multi-class SVM}

Classifying data are a common task in machine learning. Suppose some given data points each belong to one of two classes, and the goal is to decide which class a new data point will be in. In the case of SVMs used. In machine learning, SVMs (also support vector networks) are supervised learning models with associated learning algorithms that analyze data and recognize patterns, used for classification and regression analysis. Given a set of training examples, each marked as belonging to one of two categories, an SVM training algorithm builds a model that assigns new examples into one category or the other, making it a non-probabilistic binary linear classifier. An SVM model is a representation of the examples as points in space, mapped so that the examples of the separate categories are divided by a clear gap that is as wide as possible. New examples are then mapped into that same space and predicted to belong to a category based on which side of the gap they fall on.

More formally, a SVM constructs a hyperplane or set of hyperplanes in a high- or infinite dimensional space, which can be used for classification, regression, or other tasks. Intuitively, a good separation is achieved by the hyperplane that has the largest distance to the nearest training data point of any class (so-called functional margin), since in general the larger the margin the lower the generalization error of the classifier.

Whereas the original problem may be stated in a finite dimensional space, it often happens that the sets to discriminate are not linearly separable in that space. For this reason, it was proposed that the original finite dimensional space be mapped into a much higher-dimensional space, presumably making the separation easier in that space. To keep 
Table 1: Performance compraission of various methods in literature

\begin{tabular}{|c|c|c|c|c|}
\hline S.No. & Author/title & Method & $\begin{array}{l}\text { Classification } \\
\text { method }\end{array}$ & Result \\
\hline 1 & $\begin{array}{l}\text { Acree et al. (1999) - Is there a gender } \\
\text { difference in fingerprint ridge density? }\end{array}$ & On the basis of ridge density & - & - \\
\hline 2 & $\begin{array}{l}\text { Badawi et al. (2006) - Fingerprint-based } \\
\text { gender classification }\end{array}$ & $\begin{array}{l}\text { Features extracted were; ridge count, } \\
\text { RTVTR, white lines count, and ridge } \\
\text { count asymmetry, and pattern type } \\
\text { concordance. }\end{array}$ & $\begin{array}{l}\text { FCM, LDA, and } \\
\text { NN }\end{array}$ & $\begin{array}{l}\text { Results of } 80.39 \%, 86.5 \% \text {, } \\
\text { and } 88.5 \% \text { using FCM, LDA, } \\
\text { and NN, respectively }\end{array}$ \\
\hline 4 & $\begin{array}{l}\text { Wang et al. (2008) - Gender determination } \\
\text { using fingertip features }\end{array}$ & $\begin{array}{l}\text { Ridge count, ridge density, and finger } \\
\text { size }\end{array}$ & & $\begin{array}{l}86 \% \text { accuracy is obtained by } \\
\text { using ridge count and finger } \\
\text { size features together }\end{array}$ \\
\hline 5 & $\begin{array}{l}\text { Kaur et al. (2011) - Determination of } \\
\text { gender differences from fingerprint ridge } \\
\text { density in two northern Indian populations }\end{array}$ & Epidermal ridge density & & \\
\hline 6 & $\begin{array}{l}\text { Rastogi et al. - A study of fingerprints in } \\
\text { relation to gender and blood group }\end{array}$ & $\begin{array}{l}\text { Association between distribution } \\
\text { of fingerprint patterns, blood group } \\
\text { and gender }\end{array}$ & & \\
\hline 7 & $\begin{array}{l}\text { Kaur et al. (2012) - Fingerprint based } \\
\text { gender identification using frequency } \\
\text { domain analysis }\end{array}$ & Frequency domain analysis & $\begin{array}{l}\text { FFT, DCT and } \\
\text { PSD }\end{array}$ & $\begin{array}{l}\text { Results of } 90 \% \text {, and } 79.07 \% \\
\text { for female and male samples } \\
\text { respectively }\end{array}$ \\
\hline 8 & $\begin{array}{l}\text { Tom et al. (2013) - Fingerprint based } \\
\text { gender classification using 2D DWTs and } \\
\text { PCA }\end{array}$ & Frequency domain analysis & $\begin{array}{l}\text { 2D DWTs and } \\
\text { PCA }\end{array}$ & $70 \%$ \\
\hline
\end{tabular}

RTVTR: Ridge thickness to valley thickness ratio, FCM: Fuzzy C means, LDA: Linear discriminant analysis, NN: Neural network, SVM: Support vector machines, FFT: Fourier transform, DCT: Discrete cosine transform, PSD: Power spectral density, DWT: Discrete wavelet transforms, PCA: Principal component analysis, 2D: Two-dimensional

the computational load reasonable, the mappings used by SVM schemes are designed to ensure that dot products may be computed easily in terms of the variables in the original space, by defining them in terms of a kernel function $\mathrm{K}(\mathrm{x}, \mathrm{y})$ selected to suit the problem [36].

Unfortunately, unlike KDA, SVMs do not generalize naturally to the multi-class classification case. SVMs are binary classifiers, and as such, in their original form, they can only decide between two classes at once. However, many approaches have been suggested to perform multi-class classification using SVMs, one of which will be exemplified below. Suppose we have to decide between three classes, A, B, and C. Now, suppose all we have are binary classifiers, i.e., methods which can automatically decide only between two classes [37-40]. A possible approach in order to use our binary classifiers in a multi-class classification problem would be to try to divide our multi-class problem into a set of binary problems. The left matrix below shows all possible combinations of binary decision problems which can be formed by taking our three classes (Table 1).

\section{CONCLUSION}

Due to the big potential of fingerprints as an useful methodology of classification of shot has been created within the present work to research their correlation with gender of an individual. Gender identification will facilitate effectively cut back the search time by limiting the next looking stage to either male information or feminine information. Once an individual is known as male or feminine, then any appropriate biometric attribute may be used for additional classification. Identification of gender may give a vital suggest numerous security and surveillance based mostly applications.

\section{REFERENCES}

1. Vadivel M, Arulkumaran T. Gender identification from finger print images based on a supervised learning approach. IPASJ Int J Comput Sci 2014;2(7):24-8.

2. Ekanem A, Abubakar H, Dibal NI. A study of fingerprints in relation to gender and blood group among residents of Maiduguri, Nigeria. IOSR J Dent Med Sci 2014;13(8):18-20.
3. Wadhwa R, Kaur M, Singh KV. Age and gender determination from finger prints using RVA and DCT coefficients. IOSR J Eng 2013;3(8):5-9.

4. Mudrova M, Prochazka A. Principal Component Analysis In Image Processing. Institute of Chemical Technology. Czech Republic: Prague Department of Computing and Control Engineering; 2013.

5. Tom R, Arulkumaran T. Fingerprint based gender classification using 2D discrete wavelet transforms and principal component analysis. Int $\mathbf{J}$ Eng Trends Technol 2013;4(2):199-203.

6. Azad R, Azad B, Kazerooni IT. Optimized method for real-time face recognition System based on PCA and multiclass support vector machine. Adv Comput Sci Int J2013;2(5):126-32.

7. Kumar L, Agarwal S, Garg R, Pradap A, Mishra VK. Gender determination using fingerprints in the region of Uttarakhand. Indian Acad Forensic Med 2013;35(4):308-11.

8. Francis M, Koul O, Rokade P. Gender specification using touch less fingerprint recognition. Int $\mathrm{J}$ Comput Appl Technol Res 2013;2(6):717-22.

9. Desai B, Ruchi J, Prakash T, Kalyan JL. Study of fingerprint patterns in relationship with blood group and gender - A statistical review. Res J Forensic Sci 2013;1(1):15-7.

10. Raloti SK, Shah KA, Patel VC, Menat AK, Mori RN, Chaudhari NK. An effort to determine blood group and gender from pattern of finger prints. Natl J Community Med 2013;4(1):158-60.

11. Bansal N, Sheikh S, Bansal R, Pallagati S. Correlation between lip prints and finger prints in sex determination and pattern predominance in 5000 subjects. J Forensic Odontostomatol 2013;31(1):8-14.

12. Valarmathy S, Kumar PS. SVM-BDT based intelligent fingerprint authentication system using geometry approach. Int J Comput Netw Security 2012;4(1):62-7.

13. Thai LH, Hai TS, Thuy NT. Image classification using support vector machine and artificial neural network. I.J. Inf Technol Comput Sci 2012;5:32-8

14. Singh G, et al. Determination of gender differences from fingerprints ridge density in two Northern Indian population of Chandigarh Region. IOSR J Eng 2012;

15. Kaur R, Mazumdar SG. Fingerprint based gender identification using frequency domain analysis. Int J Adv Eng Technol 2012;3:295-9.

16. Ponnarasi SS, Rajaram M. Gender classification system derived from fingerprint minutiae extraction. International Conference on Recent Trends in Computational Methods Communication and Controls (ICON3C); 2012. 
17. Omidiora E, Ojo O, Yekini NA, Tubi TO. Analysis, design and implementation of human fingerprint patterns system towards age and gender determination, ridge thickness to valley thickness ratio (RTVTR) and ridge count on gender detection. Int J Adv Res Artif Intell 2012;1(2):57-63.

18. Ramotowski R, Sears V, Wakefield M. Latent print development. IOSR J Eng 2012;

19. Gnanasivam P, Muttan S. Estimation of age through fingerprints using wavelet transform and singular value decomposition. Int J Biom Bioinf 2012;6(2):58-67.

20. Purohit R, Imam SA, Beg MT. Recognizing gender with fingerprints. Int J Adv Eng Technol 2011;2(4):239-41.

21. Drochioiu G, Sandu I, Olteanu GI, Mangalagiu I. Ninhydrin-based forensic investigations. Int J Crim Invest 2011;1(1):37-58.

22. Pandey PK, Singh Y, Tripathi S. Image processing using principle component analysis. Int J Comput Appl 2011;15(4):37-40.

23. Kaur R, Garg RK. Determination of gender differences from fingerprint ridge density in two Northern Indian populations. Probl Forensic Sci 2011;85:5-10

24. Connatser RM, DePaoli G, Gardner C, Lewis L. Latent print detection by macroraman imaging. Int $\mathrm{J}$ Emerg Technol Adv Eng 2010;

25. Theodoridis S Koutroumbas K. Pattern Recognition. $4^{\text {th }}$ ed. London: Academic Press; 2009.

26. Verma M, Agarwal S. Fingerprint based male - female classification. International Workshop on Computational Intelligence in Security for Information Systems (CISIS'08). Genoa, Italy; 2008. p. 251-7.

27. Wang J, Lin CL, Chang YH, Nagurka ML, Yen CW, Yeh C. Gender determination using fingertip features. Internet $\mathrm{J}$ Med Update 2008;3(2):22-8.
28. Sinha C, Sa PK. Gender classification from facial images using PCA and SVM. Int J Emerg Technol Adv Eng 2013;

29. Gungadin S. Sex determination from fingerprint ridge density. Int J Med Update 2007;2(2):1-4.

30. Badawi A, Mahfouz MR, Tadross R, Jantz R. Fingerprint - based gender classification. The International Conference on Image Processing, Computer Vision, and Pattern Recognition; 2006.

31. Maltoni D, Maio D, Jain A, Prabhakar S. Handbook of Fingerprint Recognition. $1^{\text {st }}$ ed. New York: Springer; 2003.

32. Cristianini N, Taylor JS. An Introduction to Support Vector Machines and Other Kernel-Based Learning Methods. Cambridge: Cambridge University Press; 2000

33. Acree MA. Is there a gender difference in fingerprint ridge density? Forensic Sci Int 1999; 102:35-44.

34. Rastogi P, Pillai KR. A study of fingerprints in relation to gender and blood group. J Indian Acad Forensic Med 2010;32(1):11-14.

35. Gornale SS, Geetha CD, Kruthi R. Analysis of fingerprint image for gender classification using spatial and frequency domain analysis. Am Int J Res Sci Technol Eng Math 2013;1(1):46-50.

36. Jain NK, Sharma S, Paliwal A. A real time approach to determine the gender using fingerprints. IJAIR 2012;:229-33

37. Rai A. Attribute based level adaptive thresholding algorithm (ABLATA) for image compression and transmission. J Math Comput Sci 2014;12:211-8

38. Rai A. Artificial intelligence for emotion recognition. J Artif Intell Res Adv 2014;1(2):24-30.

39. Rai A. Air computing: A parallel computing module for offloading computational workload on neighboring android devices. Recent Trends Parallel Comput 2015;1(3):10-3.

40. Rai A. Attribute based level adaptive thresholding algorithm for object extraction. J Adv Robot 2015;1(2):64-8. 